06,11

\title{
Индуцированный фазовый переход в прозрачной керамике $\mathrm{PbMg}_{1 / 3} \mathrm{Nb}_{2 / 3} \mathrm{O}_{3}-x \mathrm{~Pb}\left(\mathrm{Zr}_{0.53} \mathrm{Ti}_{0.47}\right) \mathrm{O}_{3}$
}

\author{
(С) Л.С. Камзина \\ Физико-технический институт им. А.Ф. Иофрфе РАН, \\ Санкт-Петербург, Россия \\ E-mail: ASKam@mail.ioffe.ru
}

Поступила в Редакцию 22 января 2020 г.

В окончательной редакции 22 января 2020 г.

Принята к публикации 28 января 2020 г.

\begin{abstract}
Исследовались временны́е зависимости диэлектрической проницаемости и оптическое пропускание в прозрачной сегнетокерамике $\mathrm{Pb}\left(\mathrm{Mg}_{1 / 3} \mathrm{Nb}_{2 / 3}\right) \mathrm{O}_{3}-x \mathrm{~Pb}\left(\mathrm{Zr}_{0.53} \mathrm{Ti}_{0.47}\right) \mathrm{O}_{3}(x=16,23,33 \%)$ в электрических полях $0<E<6 \mathrm{kV} / \mathrm{cm}$. Показано, что даже в полях, меньших коэрцитивного поля, в соединениях с $x=16$ и $23 \%$ за короткий промежуток времени происходит резкое уменьшение диэлектрической проницаемости, в то время как в керамике с $x=33 \%$ эти изменения значительно меньше и происходят за более длительное время. Обнаружено, что стабильность индуцированной в поле фазы после выключения поля сильно зависит от состава исследуемых керамик: чем больше величина $x$, тем стабильнее индуцированная фаза. Наблюдаемое различие во временных зависимостях диэлектрической проницаемости и стабильность индуцированной фазы в керамиках разного состава объясняется разными размерами сегнетоэлектрических доменов.
\end{abstract}

Ключевые слова: сегнетоэлектричество, релаксоры, фазовые переходы, прозрачная керамика.

DOI: 10.21883/FTT.2020.06.49350.009

\section{1. Введение}

Свинецсодержащие твердые растворы со структурой перовскита, состоящие из сегнетоэлектрика и релаксора, привлекают все большее внимание исследователей. Они представляют не только фундаментальный, но и практический интерес, т.к. обладают великолепными диэлектрическими, пьезоэлектрическими и электрострикционными свойствами, что важно для применений. Среди свинецсодержащих твердых растворов особое место занимают керамические соединения, содержащие $\left(\mathrm{Pb}\left(\mathrm{Mg}_{1 / 3} \mathrm{Nb}_{2 / 3}\right) \mathrm{O}_{3}\right.$ or $(\mathrm{PMN})$ и $\mathrm{Pb}\left(\mathrm{Zr}_{1-x} \mathrm{Ti}_{x}\right) \mathrm{O}_{3}$ or $(\mathrm{PZT})$. Выбор таких составов является неслучайным. Эти два типа керамик обладают хорошими пьезоэлектрическими характеристиками, что в свою очередь делает каждую керамику подходящей для разных применений.

Пьезоэлектрические преобразователи на основе PZT с составами вблизи морфотропной фазовой границы (МФГ) одно из основных применений PZT керамики. Чем ближе композиция к МФГ, чем лучше пьезоэлектрические и др. свойства. Однако недостатками PZT являются высокие диэлектрические потери и большое коэрцитивное поле. Это делает PZT неудобным объектом для ряда применений.

Альтернативными материалами являются электрострикционные материалы, которые обладают большой электрострикцией и малыми коэрцитивными полями. PMN сегодня признан в качестве лучшего представителя релаксорного электрострикционного материала. PMN является релаксором, имеет низкую температуру синтеза, высокую диэлектрическую проницаемость, большую деформацию и широкий максимум диэлектрической про- ницаемости, а также низкие потери и узкую петлю гистерезиса. Это делает PMN хорошим кандидатом для ряда применений в электронике и микроэлектронике, например, в многослойных конденсаторах, сенсорах и актюаторах. Однако составы PMN имеют относительно низкий коэффициент электромеханической связи по сравнению с РZT.

Твердые растворы PMN-xPZT обладают лучшими пьезоэлектрическими и диэлектрическими свойствами, чем отдельно взятые PMN и PZT [1]. Кроме того, свойства этих твердых растворов могут меняться в широких пределах в зависимости от содержания компонент. Составы с малой величиной $x$ являются типичными релаксорами. С увеличением $x$ уменьшается доля релаксорной фазы, и соединение становится нормальным сегнетоэлектриком. Переходная область между релаксорным и нормальным сегнетоэлектрическим поведением наблюдается в составах, расположенных в композиционной области $0.40<x<0.50$. Структурный анализ, проведенный в [2], показал, что в этой композиционной области при комнатной температуре наблюдается сосуществование псевдокубической и ромбоэдрической фаз. Для $x<0.40$ составы имеют псевдокубическую симметрию и ромбоэдрическую для $0.50<x<0.70$.

В работах $[3,4]$ зучались диэлектрические свойства керамик $\mathrm{PMN}-x \mathrm{PZT}$ с разной величиной $x$. Было показано, что МФГ между тетрагональной и псевдокубической (ромбоэдрической) фазами лежит вблизи $x \sim 0.4-0.5$. Обычно фазовый переход между ромбоэдрической и тетрагональной фазами в бинарных и тройных системах типа PMN-xPT, PIN-PMN-PT и ряда других [5-7] про- 
исходит не сразу, а через промежуточную моноклинную или орторомбическую фазы.

Ситуация в соединениях PMN $-x \mathrm{PZT}$, как показано в работах $[2,8]$, существенно отличается от предыдущей. Никаких фаз (моноклинной или орторомбической) не обнаружено при МФП, а в переходной области наблюдается переход из ромбоэдрической фазы с ближним порядком в ромбоэдрическую фазу с дальним порядком. Этот переход не требует вращения поляризации. Отсутствие промежуточной фазы, и при этом наличие достаточно большого пьезоэффекта, делают соединения PMN- $x$ PZT особенно привлекательными для исследователей.

Наиболее интересным объектом для изучения является прозрачная сегнетоэлектрическая керамика PMN $-x$ PZT, т. к. при высокой прозрачности и прекрасных электрооптических свойствах она может быть использована для различных оптических применений из-за низкой стоимости продукции, простоты приготовления керамики разных размеров и форм с хорошим контролем состава.

В наших работах $[9,10]$ мы впервые приготовили прозрачную керамику $\mathrm{PMN}-x$ PZT с $x=10,16,23$ и $33 \%$ с высокой прозрачностью $(\sim 65 \%)$ и большой величиной электрооптического эффекта. Дальнейшие немногочисленные исследования оптических и диэлектрических свойств, проведенные в электрических полях $[11,12]$, показали, что доминирующим фактором в поведении этих свойствах в поле является доменная структура. Тем интереснее исследовать связь доменной структуры с другими, еще не изученными, свойствами. Совсем нет сведений о ее влиянии на кинетику процесса поляризации при индуцировании фазового перехода в электрическом поле.

Составы PMN-xPZT $(x<0.40)$ относятся к релаксорам. Напомним, что необычные свойства релаксоров связаны с полярными нанообластями (PNR), которые возникают при высоких температурах, растут при понижении температуры и замерзают ниже температуры Фогеля-Фулчера $T_{\mathrm{vf}}$, не образуя дальнего порядка (стекольное состояние). Свойства системы в таком замороженном неравновесном состоянии должны зависеть от времени, а также от поведения PNR, которое определяется составом, температурой, внешним полем и рядом других факторов. Приложение электрического поля может трансформировать релаксорное состояние в сегнетоэлектрическое упорядоченное состояние с дальним порядком. Именно такие процессы, индуцированные электрическим полем, играют важную роль в поведении релаксорных соединений и интенсивно исследуются для того, чтобы выявить природу PNR и их эволюцию под действием различных внешних условий.

В настоящей работе изучена кинетика индуцированного фазового перехода в прозрачной сегнетокерамике PMN-xPZT с разным содержанием $x(x=16,23,33 \%)$ и ее связь с доменной структурой. Измерены оптическое пропускание и временные зависимости изменения диэлектрической проницаемости в различных электрических полях.

\section{2. Исследуемые образцы и методика эксперимента}

Высокопрозрачная керамика PMN-xPZT $(x=16,23$, 33) с соотношением $\mathrm{Ti}: \mathrm{Zr}$ равным 53:47, приготовлялась с использованием необычного двухступенчатого метода спекания, описанного в нашей работе [9]. Для того чтобы увеличить вероятность образования перовскитовой фазы и уменьшить присутствие пирохлорной фазы при приготовлении керамики, вместо цирконата свинца использовался цирконат-титанат свинца.

Полученные образцы были высокого оптического качества. Для оптических измерений спеченные образцы полировались до толщины $0.5 \mathrm{~mm}$. Диэлектрические и оптические свойства этих прозрачных керамик подробно исследовались в наших работах [9-11] и в работе [12]. Образцы, изучаемые в данной работе, имели следующие характерные температуры: PMN-16PZT температура Кюри $T_{c} \sim 320 \mathrm{~K}$, температура ФогеляФулчера $T_{\mathrm{vf}}=285-295 \mathrm{~K} ; \mathrm{PMN}-23 \mathrm{PZT}-T_{c} \sim 340 \mathrm{~K}$, $T_{\mathrm{vf}}=315-320 \mathrm{~K}, \quad \mathrm{PMN}-33 \mathrm{PZT}-T_{c} \sim 380-390 \mathrm{~K}$, $T_{\mathrm{vf}} \sim 360 \mathrm{~K}$.

Универсальный измеритель Е7-11 использовался для диэлектрических измерений. Для оптических измерений применялся $\mathrm{He}-\mathrm{Ne}$-лазер.

Перед каждым измерением образец сначала нагревался без поля до температур, превышающих температуру $T_{\max \varepsilon}$ на $\sim 50 \mathrm{~K}$, и выдерживался при этой температуре 10-15 min для того, чтобы устранить в образцах эффекты памяти, связанные с приложением электрического поля. После отжига образцы охлаждались без поля до фиксированной температуры, при которой к ним прикладывалось постоянное электрическое поле разной напряженности. После приложения электрического поля осуществлялась регистрация изменения диэлектрической проницаемости со временем на частоте $1 \mathrm{kHz}$. Измерения проводились непосредственно после отжига образцов.

\section{3. Экспериментальные результаты и обсуждение}

На рис. 1 показана зависимость пропускания образцов PMN- $x$ PZT с разным содержанием $x$ от длины волны света, опубликованная нами в работе [9]. Из рисунка видно, что образцы PMN-33PZT имеют более низкую прозрачность в видимой и инфракрасной области по сравнению с образцами с меньшим $x$. Пропускание образцов PMN-16PZT и PMN-23PZT при комнатной температуре в области 600-2100 nm составляло $\sim 65 \%$, а образцов $\mathrm{PMN}-33 \mathrm{PZT} \sim 40 \%$, потери на отражение были 20-25\%. Изучение микроструктуры исследуемых керамик и наблюдение сегнетоэлектрических доменов с помощью пьезорезонансного силового микроскопа, проведенное нами в работе [9], показало, что керамики PMN-16PZT и PMN-23PZT содержат мелкие нерегулярные пальчиковые домены, в то время как 
состав PMN?33PZT характеризуется присутствием больших сегнетоэлектрических макродоменов в комбинации с небольшой долей пальчиковых доменов. Именно присутствие доменов больших размеров приводит к увеличению рассеяния света и уменьшению прозрачности.

На рис. 2-4 представлены временнб́е зависимости изменений диэлектрической проницаемости $\left(\varepsilon / \varepsilon_{0}\right)$ в разных электрических полях для составов PMN-16PZT (рис. 2), PMN-23PZT (рис. 3) и PMN-33PZT (рис. 4), где $\varepsilon_{0}$ - диэлектрическая проницаемость до приложения поля, $\varepsilon$ - диэлектрическая проницаемость в разные моменты времени. Поле прикладывалось к образцам в течении 300-4000 s., после чего выключалось. Заметим, что поле прикладывалось ко всем образцам в стекольной фазе. Поэтому измерения на рис. 3 и 4 производились при комнатной температуре, а на рис. 2 при $280 \mathrm{~K}$, т.к. в

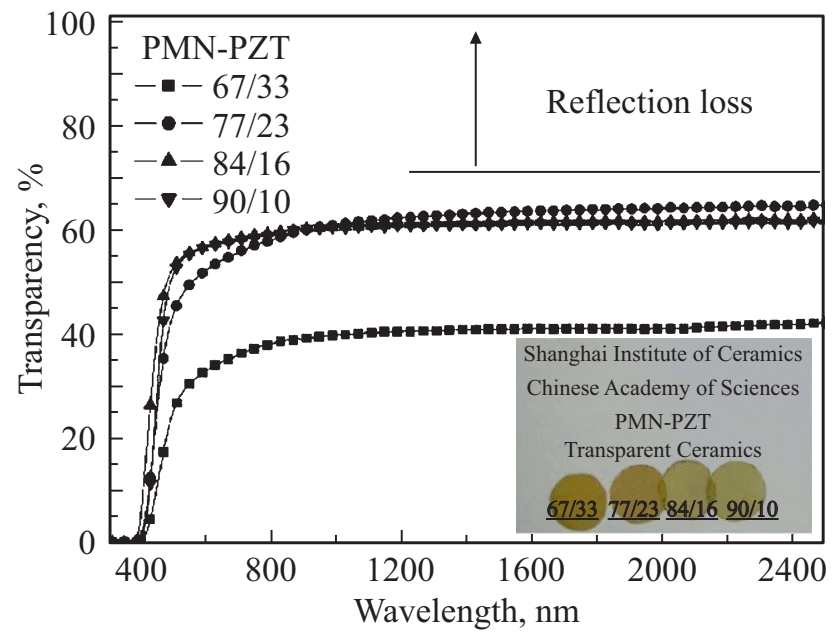

Рис. 1. Оптическое пропускание в видимой и инфракрасной областях спектра в прозрачной керамике PMN $-x$ PZT $(x=10$, $16,23,33)$ толщиной $0.5 \mathrm{~mm}$.

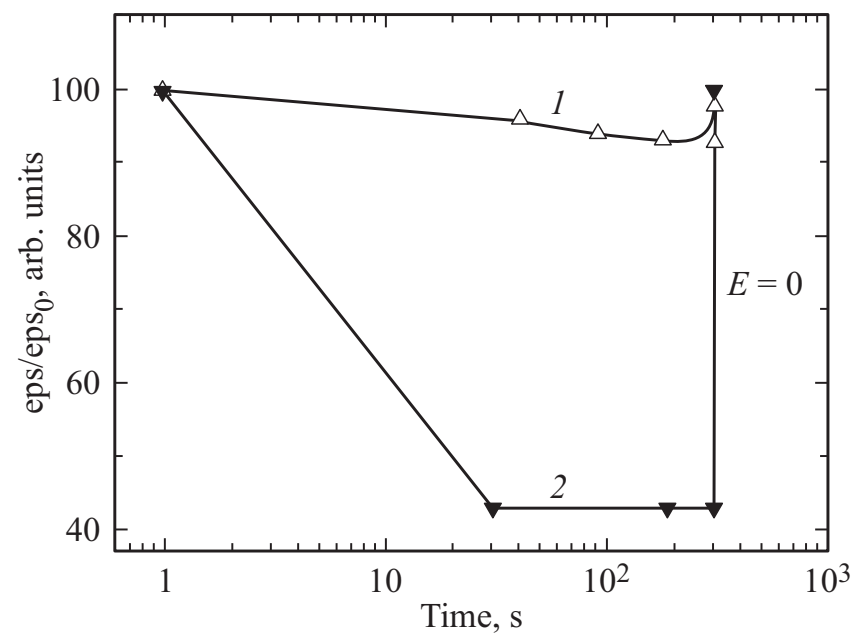

Рис. 2. Относительные изменения диэлектрической проницаемости $\left(\varepsilon / \varepsilon_{0}\right)\left(\varepsilon_{0}-\right.$ величина в нулевом поле) со временем при температуре $280 \mathrm{~K}$ в прозрачной керамике PMN-16PZT в разных электрических полях $(E, \mathrm{kV} / \mathrm{cm}): 1-1,2-3$.

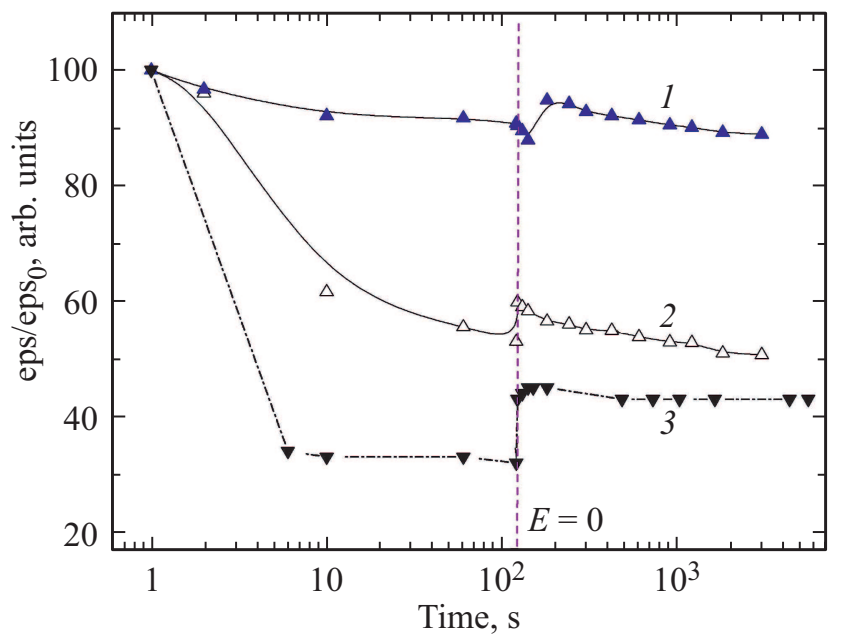

Рис. 3. Относительные изменения диэлектрической проницаемости $\left(\varepsilon / \varepsilon_{0}\right)$ со временем при температуре $300 \mathrm{~K}$ в прозрачной керамике $\mathrm{PMN}-23 \mathrm{PZT}$ в разных электрических полях $(E, \mathrm{kV} / \mathrm{cm}): 1-1,2-2,3-6$.

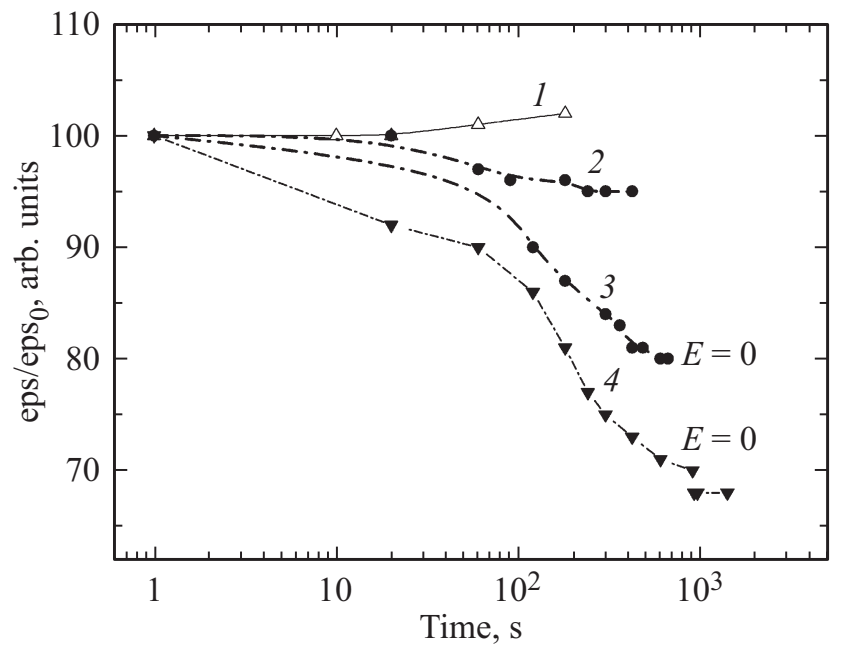

Рис. 4. Зависимость диэлектрической проницаемости $\left(\varepsilon / \varepsilon_{0}\right)$ от времени при температуре $300 \mathrm{~K}$ в прозрачной керамике $\mathrm{PMN}-33 \mathrm{PZT}$ в разных электрических полях $(E, \mathrm{kV} / \mathrm{cm})$ : $1-0.75,2-2,3-3,4-5$.

этом составе керамики температура $T_{\mathrm{vf}}$ ниже комнатной. Видно, что приложение электрического поля $\sim 1 \mathrm{kV} / \mathrm{cm}$ не приводит к существенным изменениям $\varepsilon$ во всех трех составах (кривые 1 рис. 2-4). С дальнейшим увеличением электрического поля в соединениях PMN-16PZT и PMN-23PZT за короткий промежуток времени происходит резкое уменьшение $\varepsilon$ (рис. 2 , кривая 2 и рис. 3 , кривые 2,3), в то время как в керамике PMN-33PZT эти изменения значительно меньше и происходят за более длительное время (рис. 4, кривые 2-4).

Наблюдаемое на рис. 2-4 различие в поведении $\varepsilon$ в керамиках разного состава, скорее всего, связано с разной доменной структурой. Так как в составах PMN-16PZT и PMN-23PZT домены более мелкие, то изменения диэлектрической проницаемости со 
временем происходят практически мгновенно после включения поля, превышающего пороговое (рис. 2,3). B PMN-33PZT - домены крупные, и чтобы индуцировать сегнетоэлектрическую фазу с дальним порядком нужно большее время (рис. 4). Увеличение содержания PZT компоненты приводит к уменьшению релаксорных свойств и приобретению черт нормального сегнетоэлектрика. Действительно, керамика PMN-33PZT имеет квадратную петлю гистерезиса, характерную для нормальных сегнетоэлектриков, а составы PMN-16PZT узкую ненасыщенную петлю, что свойственно релаксорам [9]. Составы PMN-23PZT занимают промежуточное положение.

Поведение индуцированной в поле фазы и ее стабильность после выключения поля сильно зависят от состава исследуемых керамик. Так в составе PMN-16PZT с наиболее выраженными релаксорными свойствами выключение электрического поля приводит к скачкообразному возрастанию величины $\varepsilon$ практически до первоначального значения (рис. 2, кривая 2). В составах PMN-23PZT после выключения электрического поля величина $\varepsilon$ (рис. 3, кривые 2,3) возрастает незначительно и ее значение меньше, чем до приложения поля. Это может быть связано с возвращением не всего объема кристалла, a только небольшой доли заполяризованных областей в релаксорное состояние. Эта частично упорядоченная сегнетоэлектрическая структура остается практически стабильной после выключения поля (рис. 3, кривая 3) во временном интервале, используемом в настоящей работе (до двух h). В составах PMN-33PZT, близких к нормальному сегнетоэлектрику, индуцированная фаза с дальним порядком остается полностью стабильной и после выключения электрического поля.

Наиболее наглядно процесс изменения $\varepsilon$ иллюстрируется рис. 5, на котором представлено изменение величины $\varepsilon / \varepsilon_{0}$ при увеличении электрического поля во всех трех составах. Каждое поле прикладывалось последовательно и выдерживалось в течение двух минут. Измерения диэлектрической проницаемости проводились спустя две минуты после приложения поля. Из кривых 1-3 видно, что приложение поля до $1 \mathrm{kV} / \mathrm{cm}$ в течение двух min изменяет величину $\varepsilon / \varepsilon_{0}$ всего на $5 \%$ во всех трех составах, в то время как приложение поля $\sim 2 \mathrm{kV} / \mathrm{cm}$ в течение того же времени приводит к существенным изменениям $\varepsilon / \varepsilon_{0}$ : в составах PMN-16PZT почти на $60 \%$ и в составах PMN-23PZT на 40\%, причем изменения происходят практически мгновенно после включения поля. Изменения величины $\varepsilon / \varepsilon_{0}$ в составах PMN-33PZT в том же поле менее существенны и составляют $\sim 5-7 \%$.

Как уже было сказано выше, уменьшение $\varepsilon$ свидетельствует об увеличении объема сегнетоэлектрической фазы. Используя данные рис. 2-5, на рис. 6 (кривые $1-3)$ представлена зависимость изменения фракции сегнетоэлектрической фазы от величины электрического поля для трех составов керамик за один и тот же промежуток времени (2 min). В составе PMN-16PZT

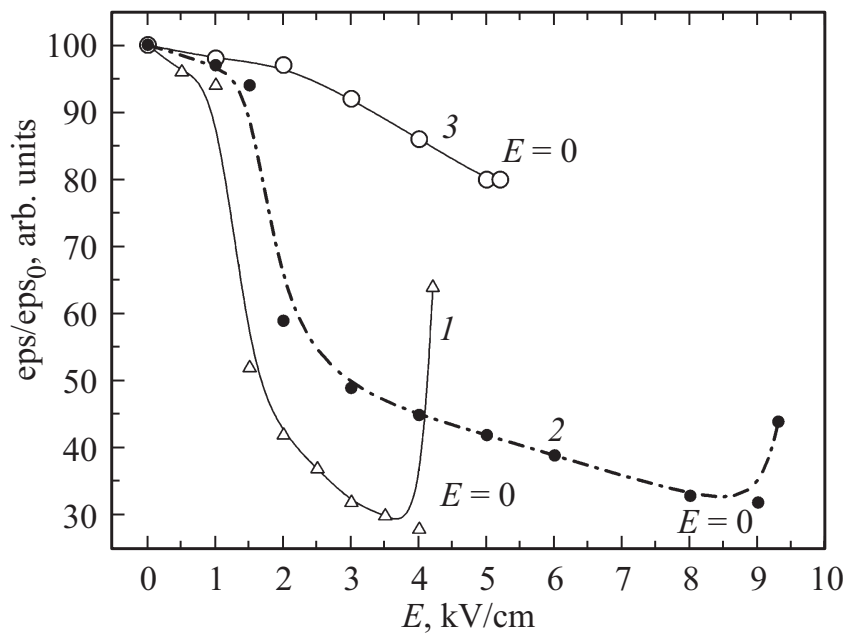

Рис. 5. Зависимость диэлектрической проницаемости от напряженности электрического поля для керамики PMN-16PZT при $275 \mathrm{~K}$ (кривая 1), PMN-23PZT при $300 \mathrm{~K}$ (кривая 2) и PMN-33PZT при $300 \mathrm{~K}$ (кривая 3). Каждое поле прикладывалось в течение $2 \mathrm{~min}$.

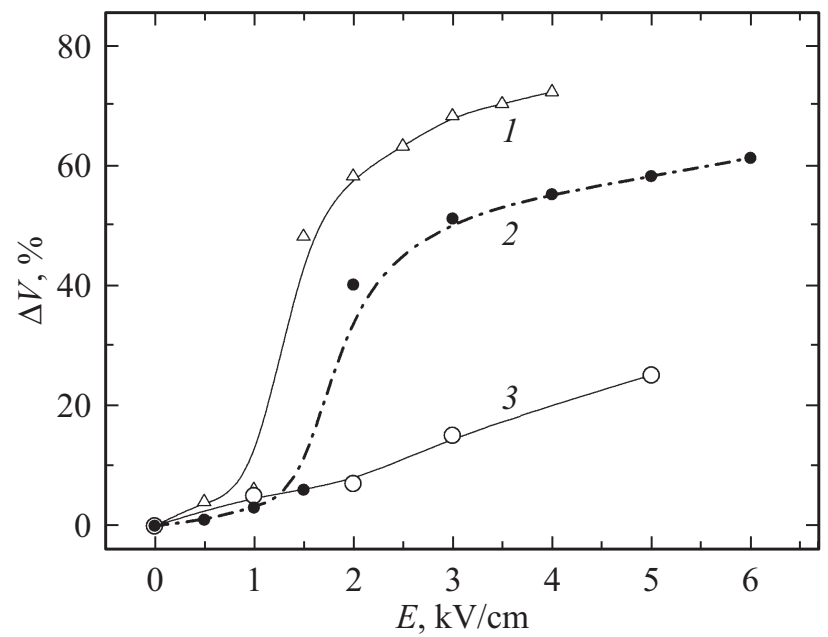

Рис. 6. Зависимость изменения фракции сегнетоэлектрической фазы от величины электрического поля для трех составов керамик за один и тот же промежуток времени (2 min): PMN-16PZT (кривая 1), PMN-23PZT (кривая 2), PMN-33PZT (кривая 3).

(рис. 6, кривая 1) доля релаксорной параэлектрической фазы велика, сегнетоэлектрические домены мелкие и даже небольшого электрического поля достаточно, чтобы индуцировать в определенной части объема сегнетоэлектрическую фазу, однако эта индуцированная фаза существует только в присутствии электрического поля (рис. 2). С увеличением содержания PZT фракция релаксорной фазы в объеме образца уменьшается, домены укрупняются, и доля сегнетоэлектрической индуцированной фазы уменьшается (рис. 6, кривые 2,3). В составе PMN-33PZT, близком к нормальному сегнетоэлектрику, фракция релаксорной фазы ничтожна, 


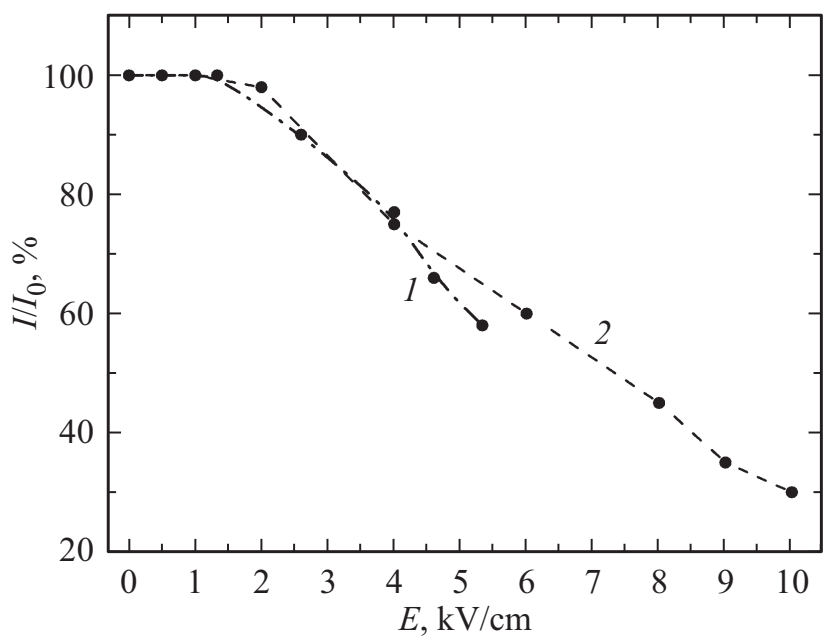

Рис. 7. Зависимость относительного изменения интенсивности оптического пропускания $\left(I / I_{0}\right)$ от напряженности электрического поля $\left(I_{0}-\right.$ первоначальное значение оптического пропускания в отсутствие электрического поля): 1 - наши измерения, 2 - данные, взятые из работы [12].

и доля, индуцированной в поле фазы, невелика $(\sim 20 \%$ в поле $5 \mathrm{kV} / \mathrm{cm})$ (кривая 3 ).

На рис. 7 (кривая 1) приведены результаты измерений оптического пропускания в электрических полях для керамики PMN-23PZT. Аналогичная зависимость наблюдалась для прозрачной керамики того же состава авторами работы [12] (кривая 2). Видно хорошее совпадение обеих кривых. Уменьшение пропускания обусловлено ростом электрически индуцированных полярных областей или доменов. Возникновение в поле частично упорядоченной сегнетоэлектрической структуры или доменов больших размеров может приводить, соответственно, к большим доменным стенкам, которые рассеивают падающий свет. Сравнивая рис. 5 и 7 можно видеть, что характер поведения диэлектрического и оптического откликов при изменении величины постоянного электрического поля идентичен. Это еще раз подтверждает преобладающий вклад доменов в пропускание прозрачной керамики. Подобные зависимости наблюдались и для керамик PMN-16PZT и PMN-33PZT.

\section{4. Заключение}

В настоящей работе изучены оптическое пропускание и изменение со временем диэлектрической проницаемости в разных электрических полях в прозрачной сегнетокерамике PMN $-x$ PZT с $x=16,23,33 \%$.

Обнаружено, что в составах с меньшим содержанием PZT (PMN-16PZT и PMN-23PZT) приложение электрического поля, большего некоторого порогового, вызывает практически мгновенное уменьшение диэлектрической проницаемости, связанное с фазовым переходом в сегнетоэлектрическую фазу с дальним порядком. $\mathrm{B}$ составах PMN-33PZT изменения диэлектрической проницаемости значительно меньше и происходят за более длительное время.

Показано, что поведение индуцированной в поле фазы и ее стабильность после выключения поля сильно зависят от состава исследуемых керамик. В керамике с наиболее ярко выраженными релаксорными свойствами (PMN-16PZT) выключение электрического поля приводит к скачкообразному возрастанию величины $\varepsilon$ до первоначального значения, наблюдаемого до приложения электрического поля. В составах, близких к нормальному сегнетоэлектрику (PMN-33PZT), индуцированная в поле фаза с дальним порядком остается полностью стабильной и после выключения электрического поля.

Наблюдаемое различие во временных зависимостях диэлектрической проницаемости в керамиках разного состава, скорее всего, связано с разными размерами сегнетоэлектрических доменов. Именно присут ствие в кубической релаксорной фазе сегнетоэлектрических доменов больших размеров (PMN-33PZT) приводит к уменьшению доли релаксорной фазы и увеличению времени, необходимого для индуцирования сегнетоэлектрической фазы с дальним порядком.

\section{Благодарности}

Автор благодарен проф. G. Li из Шанхайского института керамики за предоставление высококачественных образцов прозрачной керамики.

\section{Конфликт интересов}

У автора отсутствует конфликт интересов

\section{Список литературы}

[1] R. Yimnirun, S. Ananta, E. Meechoowas, S. Wonsaenmai. J. Phys. 36, 1615 (2003).

[2] G. Singh, V.S. Tiwari. J. Appl. Phys. 101, 014115 (2007).

[3] R. Yimnirun, S. Ananta, P. Laoratakul, S. Songklanakarin. J. Sci. Technol. 26, 529 (2004).

[4] Shujun Zhang, Sung-Min Lee, Dong-Ho Kim, Ho-Yong Lee, Thomas R. Shrout. J. Am. Ceram. Soc. 90, 3859.

[5] S.-E. Park, T.R. Shrout. J. Appl. Phys. 82, 1804 (1997).

[6] J. Kuwata, K. Uchino, S. Nomura. Ferroelectrics 37, 579 (1981).

[7] D. Vieland, J.F. Li. J. Appl. Phys. 89, 1826 (2001).

[8] Wenhui He, Tong Jiang, Qiang Li, Xiaoqing Xi, Qingfeng Yan. J. Am. Ceram. Soc. 100, 1724 (2017).

[9] W. Ruan, G.R. Li, J.T. Zeng, L.S. Kamzina, H.R. Zeng, L.Y. Zheng, A.L. Ding. J. Am. Ceram. Soc. 95, 2103 (2012).

[10] Л.С. Камзина, Wei Ruan, Guorong Li, Jiangtao Zeng. ФТT 54, 1899 (2012).

[11] Л.С. Камзина, Л.А. Кулакова, G. Li. ФТT 61, 104 (2019).

[12] Wei Zhao, Wei Ruan, Jiangtao Zeng, Lizhu Huang, Kunyu Zhao, Liaoying Zheng, Huarong Zeng, Yibo Zhou, Heji Yang, Xuezheng Ruan, Guorong Li. Appl. Phys. Lett. 104, 062907 (2014).

Редактор Т.Н. Василевская 